\title{
TEGHNIQUE, DANGERS AND SAFEGUARDS IN OSTEOTOMY OF THE SPINE
}

\author{
J. CRAWfori) ADAMS, LoNidoN, ExGLANil
}

Osteotomy of the spine, introduced by Smith-Petersen $c t$ al. in 1945, brings such profound relief to those who have become grotesquely crippled by increasing flexion deformity that if it can be done with safety it must take a secure place in orthopaedic surgical practice. The operation presents remarkably little technical difficulty. But there are serious potential dangers, both during the operation and in the early post-operative period, which demand the exercise of particular care if a successful outcome is to be reasonably assured.

\section{PRINGIPLES AND TEGHNIQUE OF OPERATION}

Smith. Petersen et al. (1945), La Chapelle (1946) and Law (1949) have described the technique of the operation, which they carry out with the patient in the prone position. I have used a modified technique, the details of which have been worked out from experiments with a model and on the cadaver, and have preferred to undertake the operation with the patient in the lateral posture, which I consider offers greater safety.

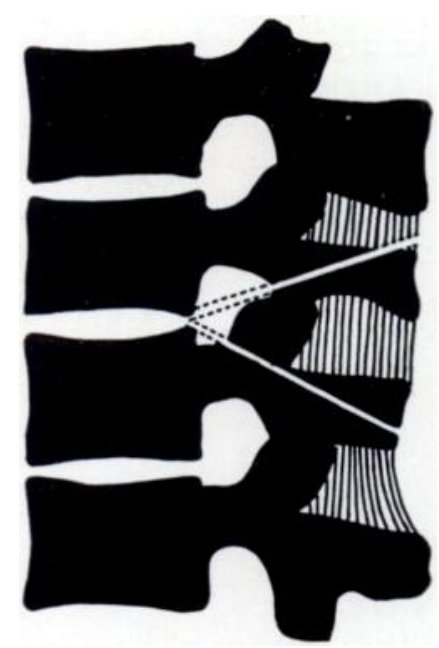

FIG. 1

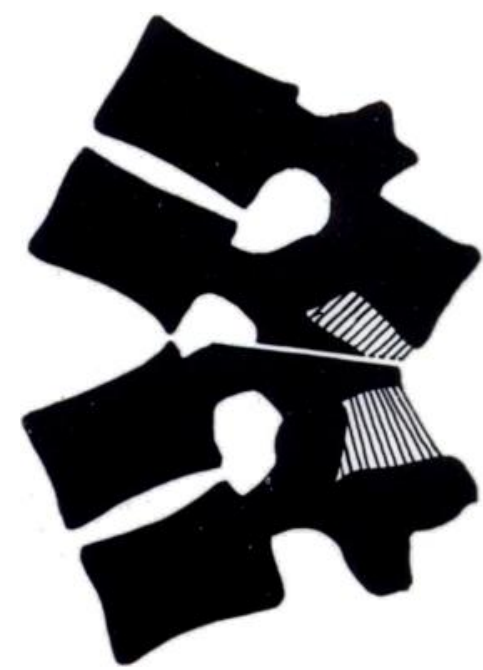

FIG. 2

Diagram showing the mechanics of spinal osteotomy. Note that the apex of the excised wedge is at the posterior margins of two adjacent vertebral bodies.

Principles--The operation consists simply in excising a wedge of bone from the posterior elements of the spinal column, after which the spine is forcibly hyperextended at the level of the osteotomy by three-point pressure-at sternum and symphysis pubis in front, and site of osteotomy behind (Figs. 1 and 2). Because of the relative fixity of the thoracic spine afforded by the attachment of the ribs, and because the cauda equina is less vulnerable than the spinal cord, the corrective operation is done at the lumbar level (usually at L.2-3 or L.3-4) wherever the principal deformity may be. The base of the excised wedge is directed posteriorly; its apex lies at the fulcrum of the corrective movement, which is at the posterior rim of the selected intervertebral disc and the margins of the adjacent vertebral bodies (or, in other words, at the anterior margin of the corresponding intervertebral foramen). The angle of 
the wedge is equal to the correction obtained, and is calculated before the operation according to the degree of deformity.

Posture-The operation is most comfortably and safely carried out with the patient in the lateral posture (preferably on his left side). The lateral posture offers several advantages, of which the following are the most important. 1) It facilitates the positioning of a grossly flexed patient on the table. 2) It lightens the inevitably difficult task of the anaesthetist. 3) It reduces the anaesthetic risk by facilitating the maintenance of a clear airway and by allowing the fullest respiratory excursion. 4) It affords greater comfort to the surgeon. 5) It makes the operation easier because any oozing blood flows away from the depths of the wound instead of into them. 6) It eliminates risk of injury to the ankylosed cervical spine by pressure of the forehead against the table. It may be argued in favour of the prone

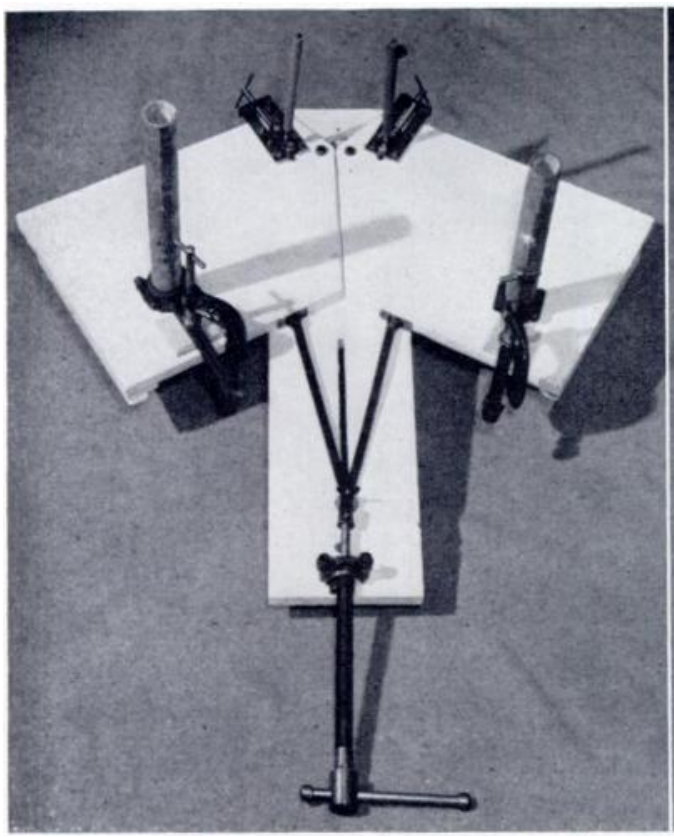

FIG. 3

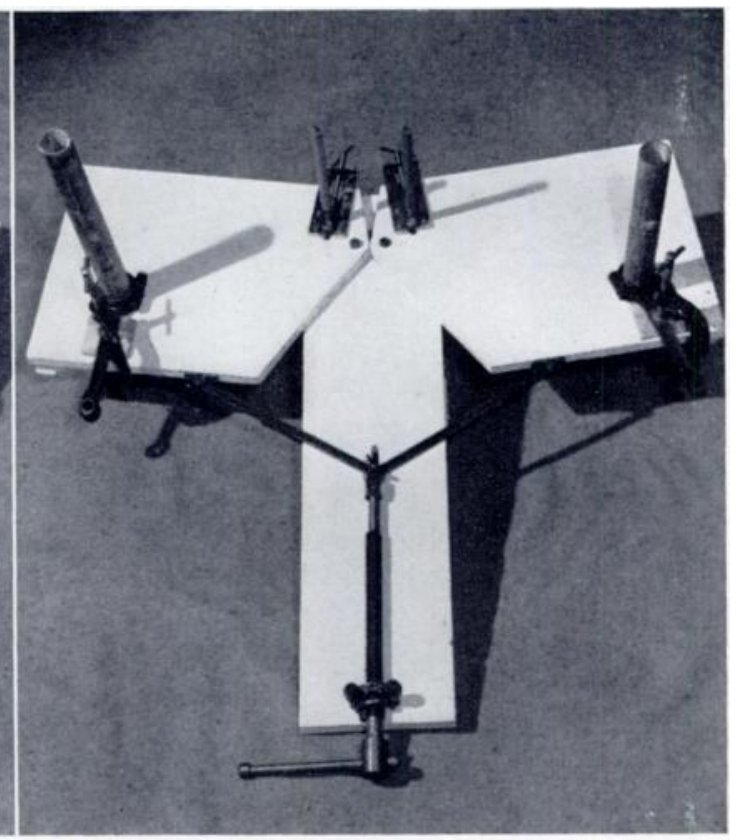

FIG. 4

Apparatus for extending the spine after osteotomy. It is shown "flexed " in Figure 3, and " extended " in Figure 4. The apparatus works by three-point pressure-at the manubrium sterni and symphysis pubis in front, and at the site of the osteotomy behind.

position that the manoeurre of extending the spine is more easily carried out in that position by "breaking" the operating table. But in practice there is little difficulty in extending the spine manually with the patient on his side. A more certain method, however, which gives the advantages of perfect control at every stage of the manoeuvre and of an uninterrupted view of the spinal theca, is provided by the use of a special extending apparatus such as that illustrated in Figures 3 and 4.* The lateral posture offers such positive advantages over the

\footnotetext{
* The apparatus consists of a divided platform, the two halves of which swivel on a cross beam. Their movement is controlled by a powerful screw with a traverse of twelve inches, which gives a range of movement of 80 degrees between the two halves of the platform. Three-point pressure is provided by vertical bars. The two anterior bars-one to bear against the manubrium sterni, the other against the sumphysis pubis - are held rigidly in position on the platform by clamps, and the position of each is easily adjustable. The posterior pressure point consists of dual bars mounted in sockets, one on each half of the platform. These sockets are on sliding members, each of which is thrust into position by a screw mechanism. (The purpose of making the søckets retractable is simply to facilitate the insertion of the bars at the final stage of the operation.) The points of pivoting and pressure are so designed that when the fulcrum bars have been drawn into position against the spinous processes above and below the incision, the axis of movement of the two halves of the platform corresponds closely with the axis of corrective movement of the spine.
} 


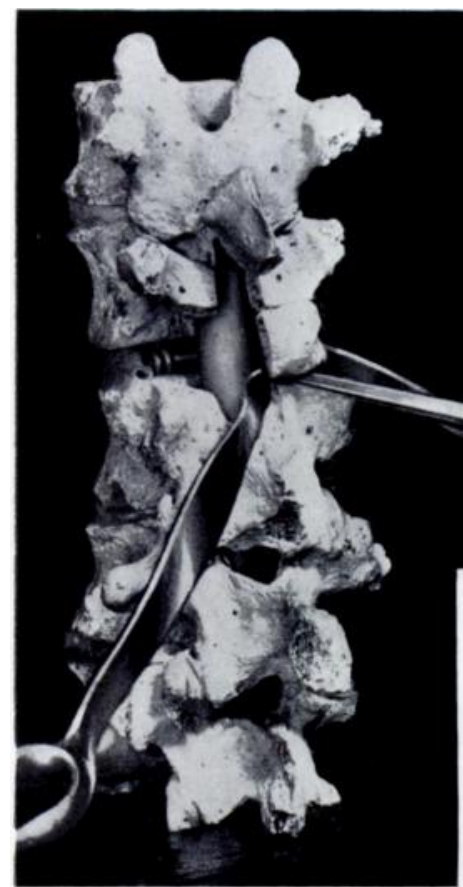

FIG. 5

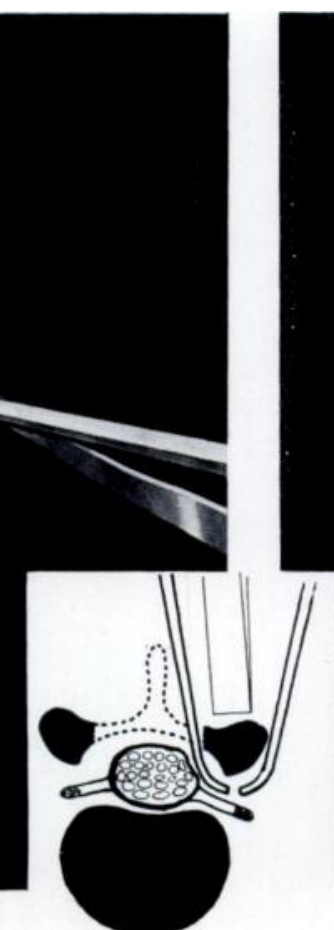

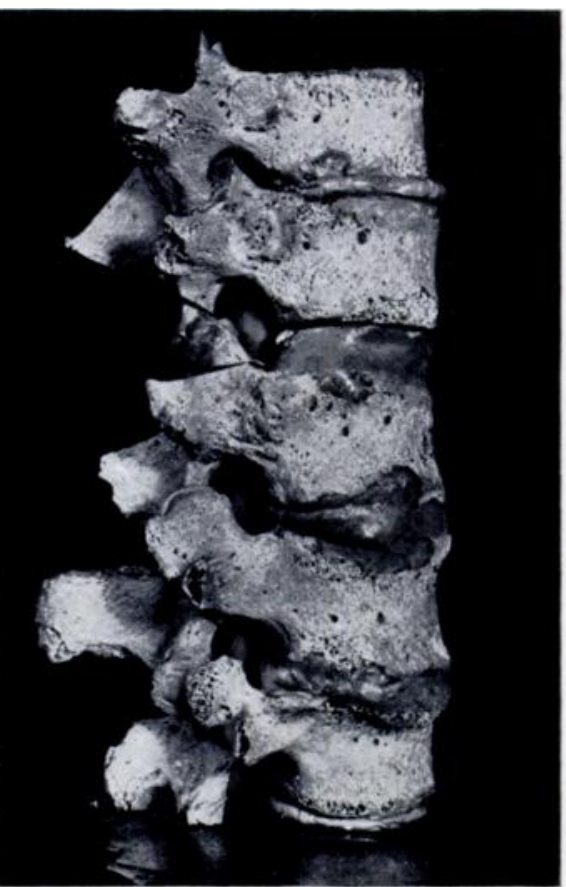

FIG. 6

Model illustrating the technique of excision of the posterior wedge. Figure 5-The spinous processes have been trimmed away and the dura has been exposed by removal of lamina and ossified ligamentum flavum. The posterior wall of the intervertebral foramen (consisting of fused facet joint and adjacent articular processes) has been excised on the left side, and is being divided on the right. Note protection of cauda equina and nerve root by curved bone levers (inset). Figure 6-Lateral view showing how the wedge to be excised tapers to its apex at the posterior margins of the vertebral bodies.

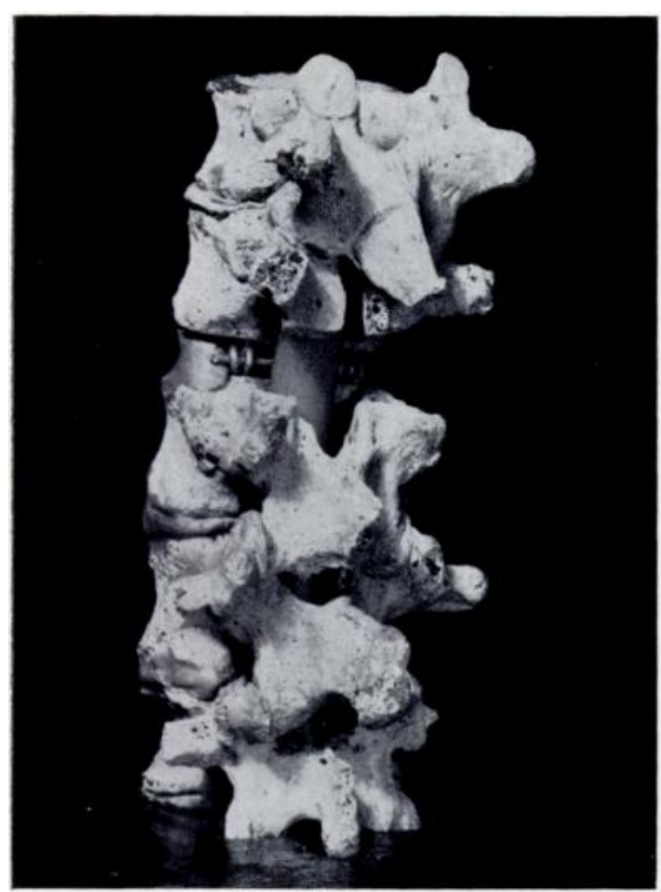

FIG. 7

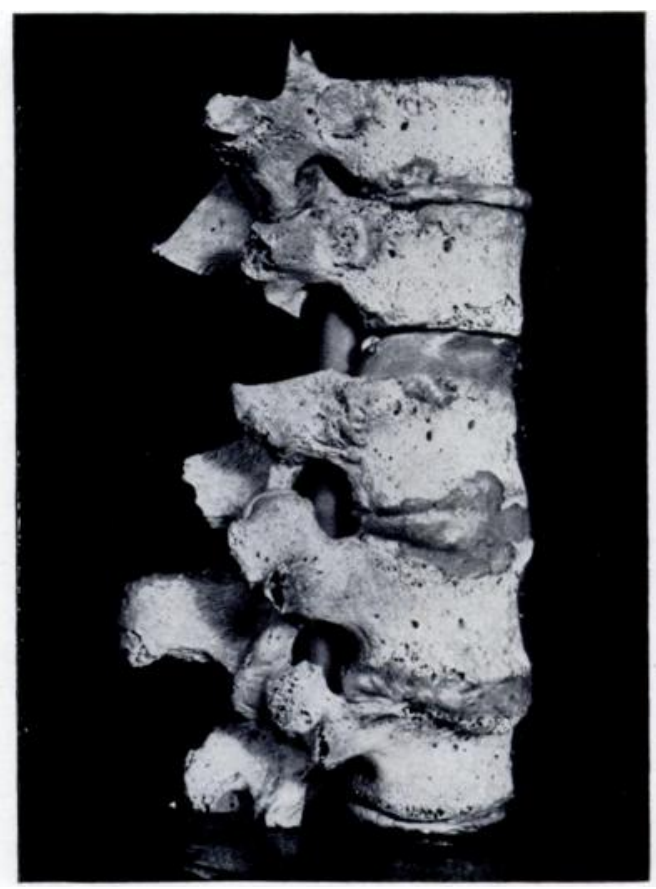

Fig. 8

Excision of the bony wedge has been completed.

THE JOURNAL OF BONE AND JOINT SURGERY 
prone that it is well worth while for any surgeon who contemplates undertaking a spinal osteotomy to acquire a simple apparatus of this type.

Technique. Positioning the patient-The patient is placed upon his left side on the platform of the spinal extension apparatus, which has been clamped to the operating table. His position on the apparatus is so adjusted that the area of the proposed incision lies between the dual fulcrum bars of the apparatus, which are then removed and sterilized in readiness for the later stage of the operation. The two leverage bars are clamped in position in contact with the manubrium sterni and the symphysis pubis respectively.

Excising the wedge-Through a midline incision the spinous processes of four vertebrae (two above and two below the site selected for correction) are exposed. Parts of two or more processes are excised according to the size of wedge to be removed, the dissection becoming narrower as it is deepened down to the plane of the laminae. The ossified ligamentum flavum and parts of adjacent laminae are excised over an appropriate length (Fig. 5), with care to avoid tearing the dura, which may be abnormally adherent to the overlying bone. When the dura has been adequately exposed attention is directed to the lateral masses of bone comprising, on each side, the superior and inferior articular processes with the intervening joint, which is usually ankylosed by bone. A block must be removed from each of these lateral masses so that the wedge-shaped defect tapers evenly to the front of the intervertebral foramen (Figs. 5 and 6). A small bone lever with a pronounced curve at its tip (such as Trethowan's) is passed through the intervertebral foramen from within the spinal canal outwards. A second lever is passed in the opposite direction to enter the foramen from the lateral side (Fig. 5). In this way the points of the two levers are made to meet within the intervertebral foramen, superficial to the emerging spinal nerve (Fig. 5, inset). The levers serve to protect the nerve and the cauda equina while a tapered block of bone, including the posterior intervertebral joint and parts of the superior and inferior processes, is removed by means of a sharp, thin osteotome (Figs. 7 and 8). This completes the removal of bone, except that the edges of the wedge-shaped defect should now be trimmed and straightened so that they will lie accurately in contact when the wedge is closed by extending the spine (Fig. 9).

The hyperextension manoeuvre-The manoeuvre of hyperextending the spine at the site of osteotomy presents no difficulty if the apparatus illustrated in Figures 3 and 4 is used. The movement is under perfect control, and the surgeon has a clear view of the spinal theca throughout. The sterilized fulcrum bars are

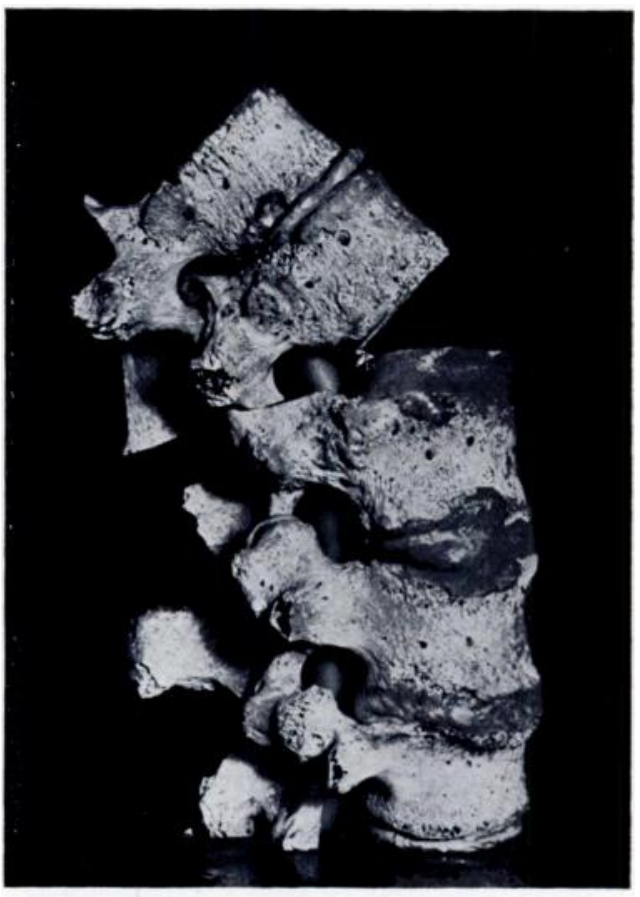

FIG. 9

The spine has been hyperextended, hinging at the posterior margins of the vertebral bodies. The raw bone surfaces are in contact. placed in their sockets on the apparatus and are thrust into position against the spine by turns of the small screws. One bar should bear against the upper segment and the other against the lower, with the incision between. The axis of movement of the apparatus then coincides automatically with the axis of corrective movement of the spine. All that is now necessary is for an assistant to turn the main screw. Closure of the wedge is best effected slowly, to avoid sudden stretching of the abdominal structures. When the wedge is closed 
and its margins meet (Fig. 9) bone chips are packed about the line of contact and the wound is closed.

If an extension apparatus is not available correction can be accomplished manually with the help of an assistant. Three-point pressure is exerted-at the site of the osteotomy behind, and at the manubrium sterni and symphysis pubis in front.

Post-operative treatment-A posterior plaster shell is constructed at the conclusion of the operation. There is no difficulty in making the shell with the patient still in the lateral posture, while full correction is held by the apparatus. When the shell is complete, the fulcrum bars are released and the patient is turned on to his back in the shell. An anterior turning case is made, preferably forthwith, or else a few hours later. The patient is turned within twentyfour hours, and the opportunity is used for finishing off, lining and mounting the plaster shell. Thereafter the patient is turned as often as required-fairly frequently during the first week, so that the condition of the skin may be closely watched.

After eight to ten weeks in the plaster bed the patient is allowed up in a full-length plaster jacket. This may usually be changed for a spinal brace three to four months after the operation, provided radiographs show satisfactory consolidation.

\section{DANGERS AND SAFEGUARDS}

Anaesthetic difficulties--In severe deformities the head may be so flexed towards the sternum that the establishment of a satisfactory airway may be difficult. The respiratory excursion is always poor. The advantages of the lateral position in diminishing these difficulties have already been mentioned.

Cauda equina injury-This is an obvious potential danger. Injury may occur either from stretching or from displacement of one spinal segment on the other. Stretching-If the posterior bone wedge is excised in the way described, the cauda equina is not subjected
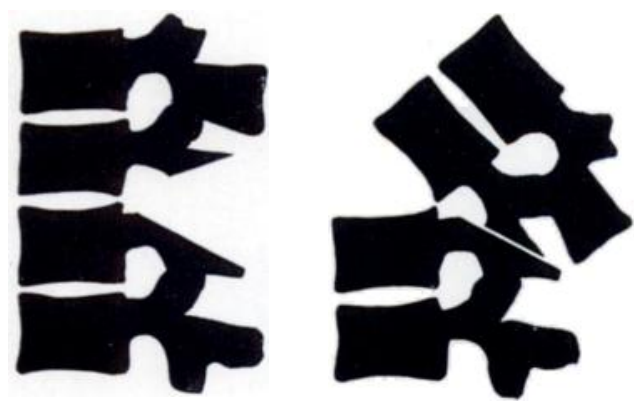

FiG. 10

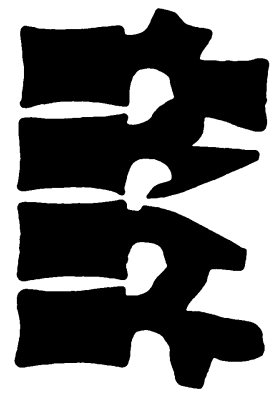

FIG. 1

Figure 10-Correct excision of posterior wedge. Its apex is at the anterior border of the intervertebral foramen. During hyperextension the fulcrum of movement remains in front of the cauda equina, which is consequently relaxed. The vertebral bodies remain in contact at their posterior margins. Figure $11-$ Incorrect excision of posterior wedge. Its apex is at the posterior border of the intervertebral foramen. l)uring hyperextension the fulcrum of movement is behind the cauda equina, which is consequently strained. The vertebral bodies are forced widely apart.

to a stretching force during the hyperextension manoeuvre. On the contrary it is relaxed, because the fulcrum of movement is in front of the spinal canal (Fig. 10). But if the apex of the excised wedge falls short of the posterior vertebral margins, or if the wedge is not cut accurately with straight sides, the fulcrum of movement may lie behind the spinal canal, and the cauda equina will consequently be subjected to a stretching force when the spine is hyperextended (Fig. 11). The safeguard is accurate excision of the wedge as already described. Displacement of one spinal segment on the other, with trapping of the cauda equina, might occur during the manoeuvre of hyperextending the spine, or during careless handling of the patient after the operation. Nevertheless if proper care is taken the risk of 
such accidents is slight. The following safeguards are suggested. 1) The sides of the excised wedge of bone must be shaped accurately so that when the wedge is closed there will be a large area of bone-to-bone contact, which helps to give stability. 2) The upper and lower segments must be under perfect control during the corrective manoeuvre, and the correction should be performed slowly and deliberately while the spinal theca is kept under direct vision. This is assured with greatest certainty by the use of a special screw extension apparatus. 3) A full length plaster shell should be moulded on to the patient at the conclusion of the operation before he is turned. 4) An accurately fitting anterior shell should be made to allow the patient to be turned without risk of producing displacement.

Rupture of the aorta or inferior vena cava-This is theoretically a distinct danger if correction through a large angle is attempted, and especially if the aorta is the seat of degenerative changes. Nevertheless one of the writer's cases has given grounds for the belief that the danger of rupture is small. The patient, a man of sixty-seven years with grotesquely severe deformity, was hyperextended at the second lumbar interspace through 90 degrees. Despite advanced atheromatous degeneration, with well marked calcification seen radiographically in the aorta, the great vessels were undamaged. Post-operative radiographs showed the calcified aorta markedly flattened antero-posteriorly. Safeguards against accidental rupture of important vessels are: 1) to carry out the extension manoeuvre very slowly, and 2) to avoid correction through a large angle-60 degrees is perhaps a safe figure-especially if degenerative aortic disease is suspected; if greater correction is needed, it would be safer done in two stages.

Fracture of an ankylosed cervical spine-If a patient with severe flexion deformity and rigid ankylosis of the cervical spine is operated upon in the prone position, great care is needed to ensure that the head is kept clear of the table and does not take any of the body weight. Otherwise when the extension force is applied to the spine, the neck may be fractured or dislocated, as in a case reported by Law (1951).* This danger does not arise if the operation is carried out with the patient in the lateral posture.

Acute dilatation of the stomach and paralytic ileus-This is the most serious danger in the early post-operative period, and probably the greatest hazard of the operation. As the lumbar spine is extended the abdominal wall is put tightly on the stretch, and the abdominal cavity with its contents is compressed antero-posteriorly. An indication of the degree of stretching to which the abdominal wall is subjected is the obliteration of the many transverse abdominal skin creases that have usually been present for many years, and the tightness evident on palpation. The exact mechanism by which acute dilatation of the stomach or paralytic ileus is produced is uncertain, but it is clear that mechanical factors are important. The condition is evidently allied to the similar complication that may follow immobilisation of the spine in a hyperextension plaster jacket, or even in an ordinary plaster hip spica, plaster bed or frame. In some cases of acute dilatation of the stomach, as distinct from ileus with distension, I believe that tensing of the superior mesenteric artery across the third part of the duodenum is an important factor, especially if the coils of small intestine are empty and hang like a weight over the brim of the pelvis, suspended on a band of tissue of which the artery is the main component. I have operated upon a patient with spontaneous high intestinal obstruction in which this was the cause and the findings were confirmed at subsequent post-mortem examination. If such a mechanism is a factor in producing obstructive symptoms after osteotomy of the spine, it is clear that the prone position will be of value both in prevention and treatment.

Whatever may be the underlying mechanism, the necessity for active treatment at the first onset of symptoms is clear. Gastric suction, intermittent or continuous, should be instituted, intravenous fluids given and the patient turned into the prone position. In my

* Surgical Treatment of the Rheumatic Diseases, published in this Number of the Journal.

vol. $34 \mathrm{~B}$, พO. 2 , MAY 1952

$\mathrm{E}$ 


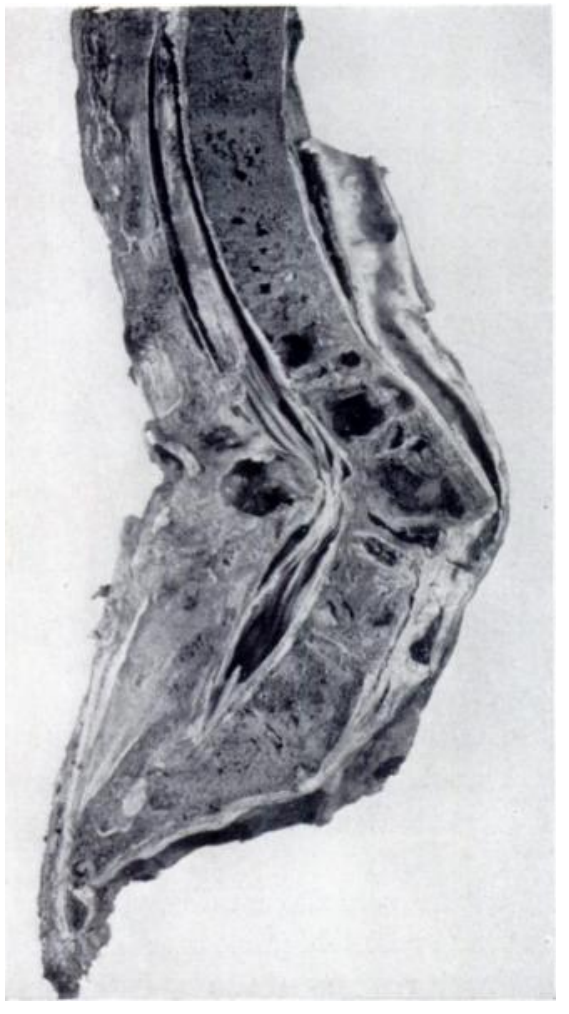

Fig. 12

specimen removed at post-mortem from a patient aged sixty-seven years who died ten weeks after spinal osteotomy. Infection originating in a pressure sore had extended forwards and given rise to a large anterior spinal abscess. last three cases I have introduced a Rvle's stomach tube through the nose before operation, and intermittent suction has been begun immediately if the patient has felt any nausea. Further trial with this prophylactic method seems justified. The importance of keeping a close watch for the development of this potentially lethal complication, and the urgent necessity for immediate and energetic counter-measures, must be emphasised.

Pressure sores--Pressure sores develop very easily in these patients. The risk is increased by the damp plaster bed, which is necessarily made at the time of the operation because of the change that is made in the shape of the spine: a hinged plaster bed is not satisfactory because it tends to produce local pressure at the site of angulation. It is of the utmost importance to avoid pressure sores, as witness one of my patients who died from an anterior vertebral abscess which was found at post-mortem to have extended directly from a deep pressure sore near the site of correction (Fig. 1:2). The following safeguards have proved effective in subsequent cases. An anterior shell or "turning case " is constructed immediately after the operation or at the latest within a few hours. This is dried out and carefully lined during the night after operation. The next day the patient is turned into the anterior shell to allow the skin of the back to dry thoroughly, and the posterior shell to be dried out, smoothed and lined. Subsequently

the skin of the back is inspected at intervals of a few days during the first two weeks, and after that at weekly or two-weekly intervals.

\section{SUMMARY}

1. The steps of the operation of spinal osteotomy, as carried out with the patient in the lateral posture, are described.

2 . Details of post-operative management are given.

3. The major risks of the operation are discussed and precautions suggested.

I am grateful to I) (ardew, of the Photographic Department of St Mary's Hospital Medical School, for the photographs.

\section{REFERENCES}

Chapelle, F. H. IA (1946): Osteotomy of the Lumbar Spine for Correction of hyphosis in a Case of Ankylosing Spondylarthritis. Journal of Bone and Joint Surgery, 28, 851.

LAw, A. W. (1949): Part II of Ankylosing Spondylitis, by Hernaman-Johnson and Law. London: Butterworth \& Co. (Publishers) Ltd.

L.Aw, A. W. (1951): Surgical Treatment of the Rheumatic Diseases. Paper read at the Annual Meeting of the British Orthopaedic Association in Edinburgh, October 1951. Published in this Number of the Journal. Simith-Petersex, M. N., LARSEN, C. B., and AUfraic, O. E. (1945): Osteotomy of the Spine for Correction of Flexion Deformity in Rheumatoid Arthritis. Journal of Bone and Joint Surgery, 27, 1. 